TM-1485

\title{
Tests of Cold Helium Compressors at Fermilab*
}

\author{
T.J. Peterson and J.D. Fuerst \\ Fermi National Accelerator Laboratory \\ P.O. Box 500, Batavia, Illinois 60510
}

October 1987

*Presented at the Cryogenic Engineering Conference/International Cryogenic Materials Conference, St. Charles, Illinois, June 14-18, 1987 


\title{
TESTS OF COLD HELIUM COMPRESSORS AT FERMILAB
}

\author{
T.J. Peterson and J.D. Fuerst \\ Fermi National Accelerator Laboratory* \\ Batavia, Illinois
}

\begin{abstract}
Fermilab has tested two cold helium compressors for possible installation in the satellite refrigerator buildings of the Tevatron cryogenic system. Operating conditions required to obtain an overall Tevatron energy upgrade from 900 to $1000 \mathrm{GeV}$ are (for each of 24 machines): $52 \mathrm{~g} / \mathrm{s}$ mass flow rate, $0.7 \mathrm{~atm}$ inlet pressure, $1.4 \mathrm{~atm}$ exhaust pressure. Acceptable efficiency is in the $60 \%$ range. Both Creare, Inc. and Cryogenic Consultants, Inc. (CCI) have supplied units for evaluation. The Creare machine is a high speed centrifugal pump/compressor which yielded $60 \%$ adiabatic efficiency (including an approximately 20 watt heat leak) with a $1.0 \mathrm{~atm}$ inlet pressure and $55 \mathrm{~g} / \mathrm{s}$ flow rate. Certain mechanical difficulties were present, chiefly the device's inability to withstand two-phase flow. CCI supplied a reciprocating unit which, after initial testing and modification, achieved $59 \%$ efficiency with an approximate 35 watt heat leak at a 0.7 atm inlet pressure and $48 \mathrm{~g} / \mathrm{s}$ flow rate. Although the device lacks the smooth, quiet operating characteristics of a turbomachine, it has endured mechanically throughout testing and is entirely insensitive to two-phase flow.
\end{abstract}

\section{INTRODUCTION}

The beam energy of Fermilab's Tevatron is presently limited to about $900 \mathrm{GeV}$ by dipole magnetic field strength. Operational data and dipole magnet quench data indicate that, in order to reliably increase the energy of the Tevatron from $900 \mathrm{GeV}$ to $1000 \mathrm{GeV}$, superconducting magnet temperatures have to be lowered by about $0.5 \mathrm{~K}$. This requires a pressure decrease of the boiling helium in the "two-phase" channel of the magnet strings. Calculations and computer simulations performed by Fermilab and by Air Products, Inc., have indicated that a cold compressor located on the satellite refrigerator two-phase return line and pumping either saturated helium vapor or two-phase helium may be the most cost-effective means to attain lower temperatures (see Fig. 1). Twenty-four cold compressors (one in each satellite refrigerator) each pumping approximately $52 \mathrm{~g} / \mathrm{s}$ of helium from $0.7 \mathrm{~atm}$ (saturated vapor or two-phase) to about $1.4 \mathrm{~atm}$ would be required. A program of testing cold compressors was begun to determine

*Operated by Universities Research Association, Inc. under contract with the U.S. Department of Energy. 


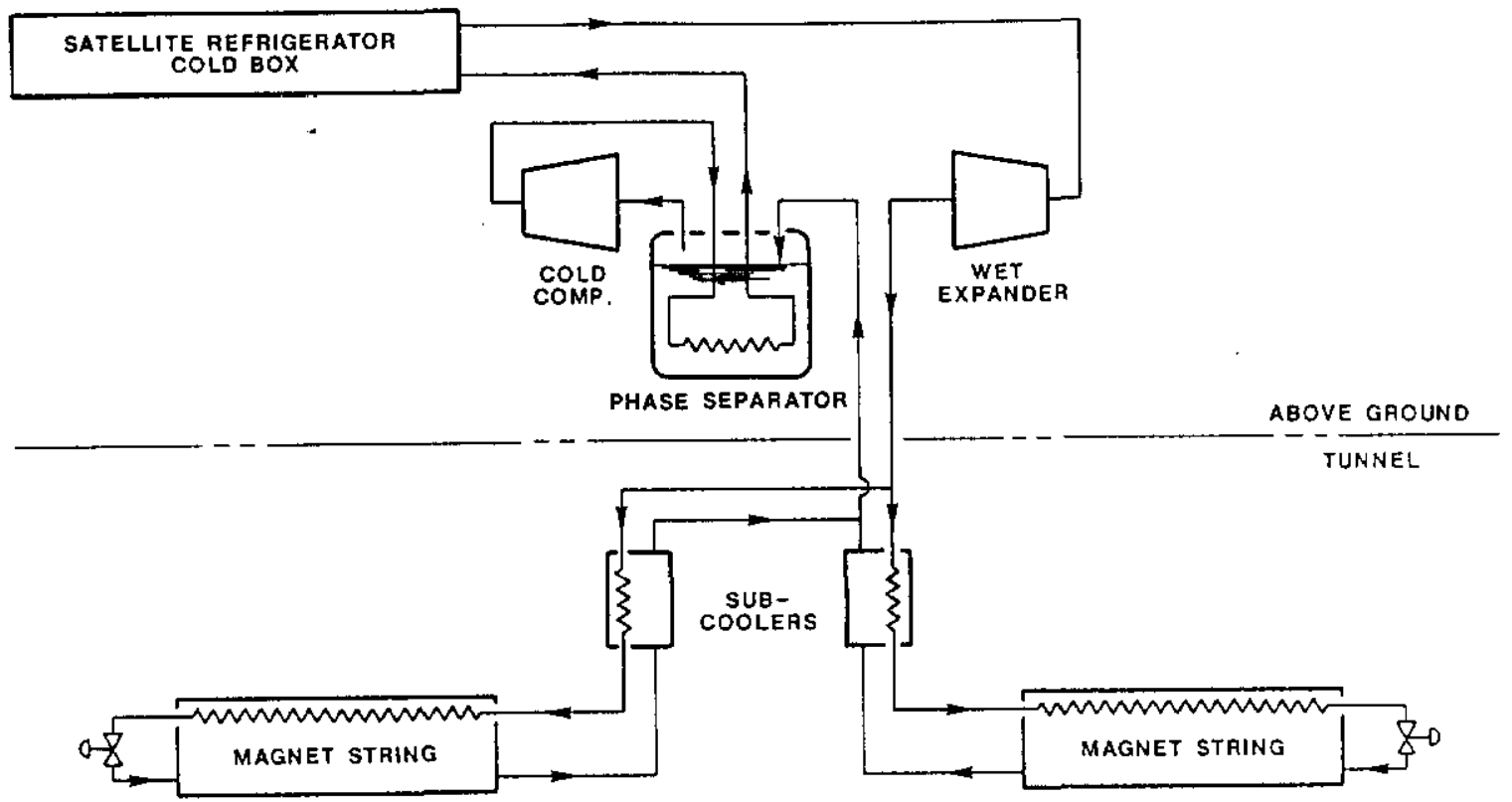

Fig. 1. Satellite refrigerator with cold compressor.

which, if any, might be suitable for use in our satellite refrigerators. Requirements include $60 \%$ minimum efficiency under design conditions and extreme reliability. The Tevatron typically runs for six months without scheduled maintenance, and all 24 compressors must be operational to provide $1000 \mathrm{GeV}$ capability.

Two cold compressors have been tested at Fermilab over the past 18 months: a centrifugal unit designed and manufactured by Creare, Inc. and a reciprocating unit designed and manufactured by Cryogenic Consultants, Inc. (CCI). Each was loaned to Fermilab for testing at helium temperatures. A brief test description follows.

\section{TEST METHOD}

Figure 2 illustrates the test configuration for the cold compressor tests. Mass flow was controlled by varying wet expander speed and was determined via a flat plate orifice meter (FI4). Instrumentation consisted of pressure taps (PI's), vapor pressure thermometers (TI's), and carbon resistors (TR's). A differential pressure type liquid level gauge, superconducting liquid level indicator, and resistance type dewar heater were also included. In addition to the standard refrigerator instrumentation, each compressor was fitted with a pressure tap, carbon resistor, and vapor pressure thermometer at both inlet and exhaust locations. Zero offsets were periodically obtained for these devices as testing progressed to ensure accuracy. Instrumentation readbacks were processed by Fermilab's existing instrumentation and control system ${ }^{12}$ which permitted real-time graphical displays and hardcopies of relevant parameters.

Methodical accumulation of data was obtained from each machine through selective manipulation of one parameter (for instance, mass flow) while maintaining control over other parameters such as compressor speed. In this manner a data map blanketing the expected range of operating conditions was created for each compressor. 


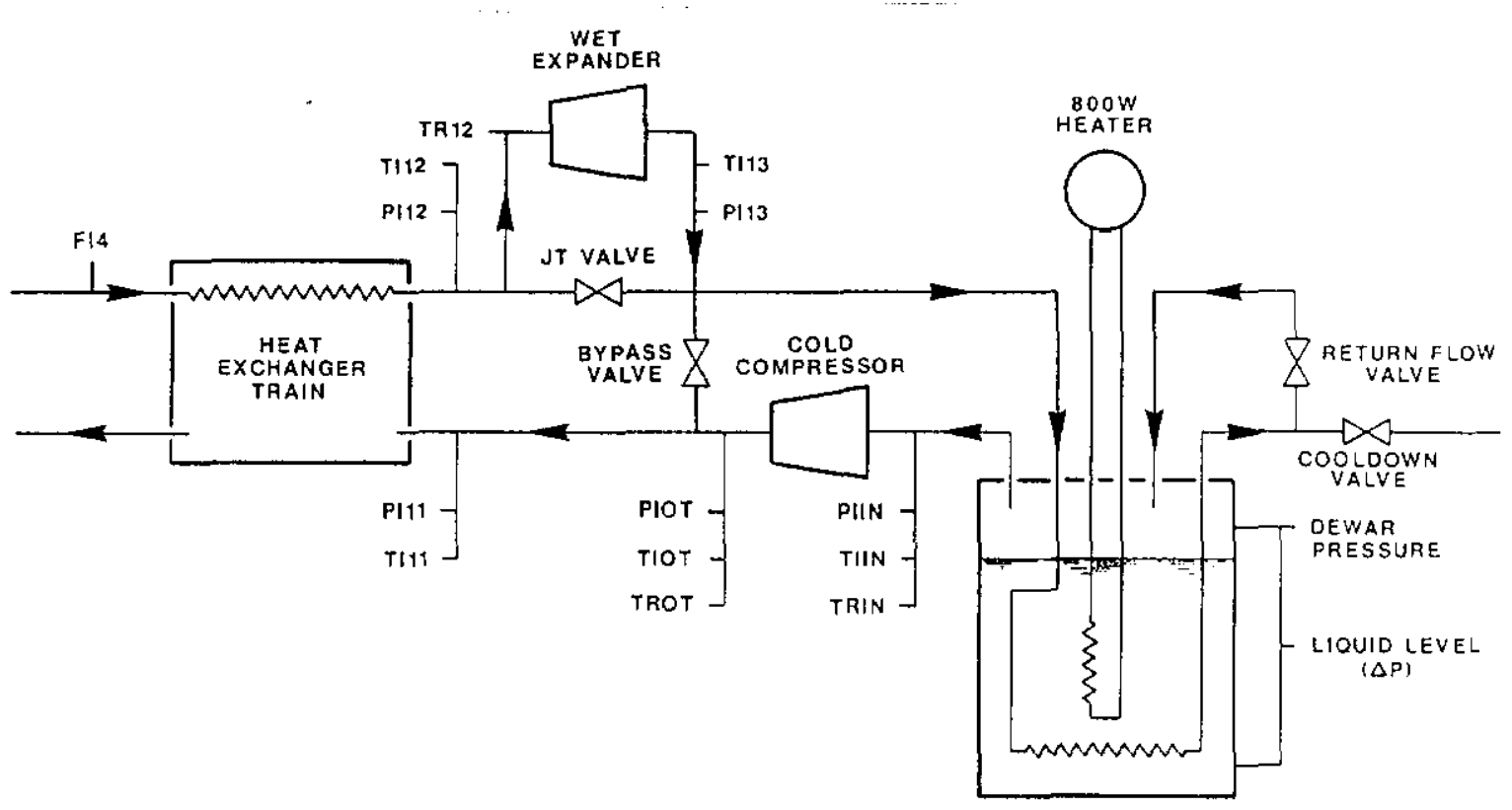

Fig. 2. Cold compressor test configuration.

\section{CREARE COLD COMPRESSOR}

\section{Description}

A generic pump/compressor has been developed by Creare and was described at the previous Cryogenic Engineering Conference. ${ }^{3}$ It is a highspeed (up to $100,000 \mathrm{rpm}$ ) electric motor driven centrifugal machine with dynamic gas radial bearings and a permanent magnetic thrust bearing. This cold compressor was tested at Fermilab in January 1986 and again in September 1986 with a different impeller. The results of the January 1986 test are described in a paper by W.D. Stacy. ${ }^{4}$ Since in January the cold compressor was operating with an impeller designed for Brookhaven's application, ${ }^{5}$ our operating conditions put it thermodynamically far from design conditions. Therefore efficiencies were expectedly low. For the tests in September (described below) the impeller was designed for a $1.0 \mathrm{~atm}$, saturated vapor inlet, $1.4 \mathrm{~atm}$ exhaust, and $55 \mathrm{~g} / \mathrm{s}$ flow. These conditions were given to Creare before a subatmospheric $(0.7 \mathrm{~atm})$ inlet condition was considered feasible.

\section{Test Results}

Table 1 summarizes the range of test conditions while Table 2 lists some typical data points. Efficiency at design conditions was $60 \%$ (with the addition of a tentative heat leak estimate of $20 \mathrm{~W} \pm 5 \mathrm{~W}$ ). Figure 3 illustrates useful head (isentropic enthalpy change) as a function of flow rate and efficiency vs. pressure ratio. The unit performed well during the two days of data accumulation (the unit was shut off each night). However, during startup on the third day the machine "buzzed" twice for a fraction of a second each time. Compressor speed momentarily decreased but operation continued. An ongoing computer plot of dewar liquid level showed that, although the dewar was about $2 / 3$ full, the liquid level readback (a 
delta-p measurement) had oscillated wildly during startup. Apparently the rapid boiling caused by the drop in dewar pressure threw liquid into the intake pipe, damaging the bearings as the wheel was hit with the liquid slug. When the cold compressor was later shut off it could not be restarted. Disassembly revealed that the impeller wheel had touched the shroud and that the gas bearings had rubbed. Although only slight polish marks were visible, this apparently resulted in our inability to run the cold compressor again. This terminated our testing in September.

Table 1. Creare Cold Compressor Test Conditions

Speed range tested:

Mass flows in data:

Pressure ratios:

Inlet pressures:

Adiabatic efficiency range: $37 \%$ to $60 \%$
50,$000 ; 66,000 ;$ and $80,000 \mathrm{rpm}$

41 to $80 \mathrm{~g} / \mathrm{s}$

1.2 to 1.5

1.00 to $1.15 \mathrm{~atm}$

$67 \%$ to $60 \%$

Table 2. Creare Cold Compressor: Four Representative Test Results

$\begin{array}{lcccc}\text { Date } & 9.25 .86 & 9.24 .86 & 9.24 .86 & 9.25 .86 \\ \text { Time } & 14: 55 & 17: 30 & 15: 51 & 12: 00 \\ \text { Inlet temp., K (TRIN) } & 4.464 & 4.426 & 4.418 & 4.373 \\ \text { Inlet temp., K (TIIN) } & 4.489 & 4.439 & 4.381 & 4.375 \\ \text { Inlet press., atm. abs. } & 1.103 & 1.089 & 1.056 & 1.042 \\ \text { Inlet enthalpy, J/g } & 31.52 & 31.18 & 31.29 & 31.16 \\ \text { Inlet entropy, J/gK } & 8.48 & 8.41 & 8.48 & 8.46 \\ \text { Exhaust temp., K (TROT) } & 5.469 & 4.970 & 5.507 & 5.263 \\ \text { Exhaust temp., K (TIOT) } & -5.4 & 4.965 & \sim 5.4 & \sim 5.3 \\ \text { Exhaust press., atm. abs. } & 1.610 & 1.292 & 1.458 & 1.451 \\ \text { Ideal exhaust enthalpy, J/g } & 34.28 & 32.22 & 33.54 & 33.37 \\ \text { Exhaust enthalpy, J/g } & 36.02 & 34.22 & 37.27 & 35.54 \\ \text { Mass flow, g/s } & 54.7 & 50.0 & 74.2 & 60.4 \\ \text { Flow, liters/s } & 3.24 & 2.94 & 4.45 & 3.68 \\ \text { Useful head, J/g } & 2.76 & 1.04 & 2.25 & 2.21 \\ \text { Comp. speed, krpm } & 66 & 50 & 80 & 66 \\ \text { Adiabatic efficiency, \% } & 61.3 & 34.2 & 37.6 & 50.5\end{array}$

a Gas bulb region 

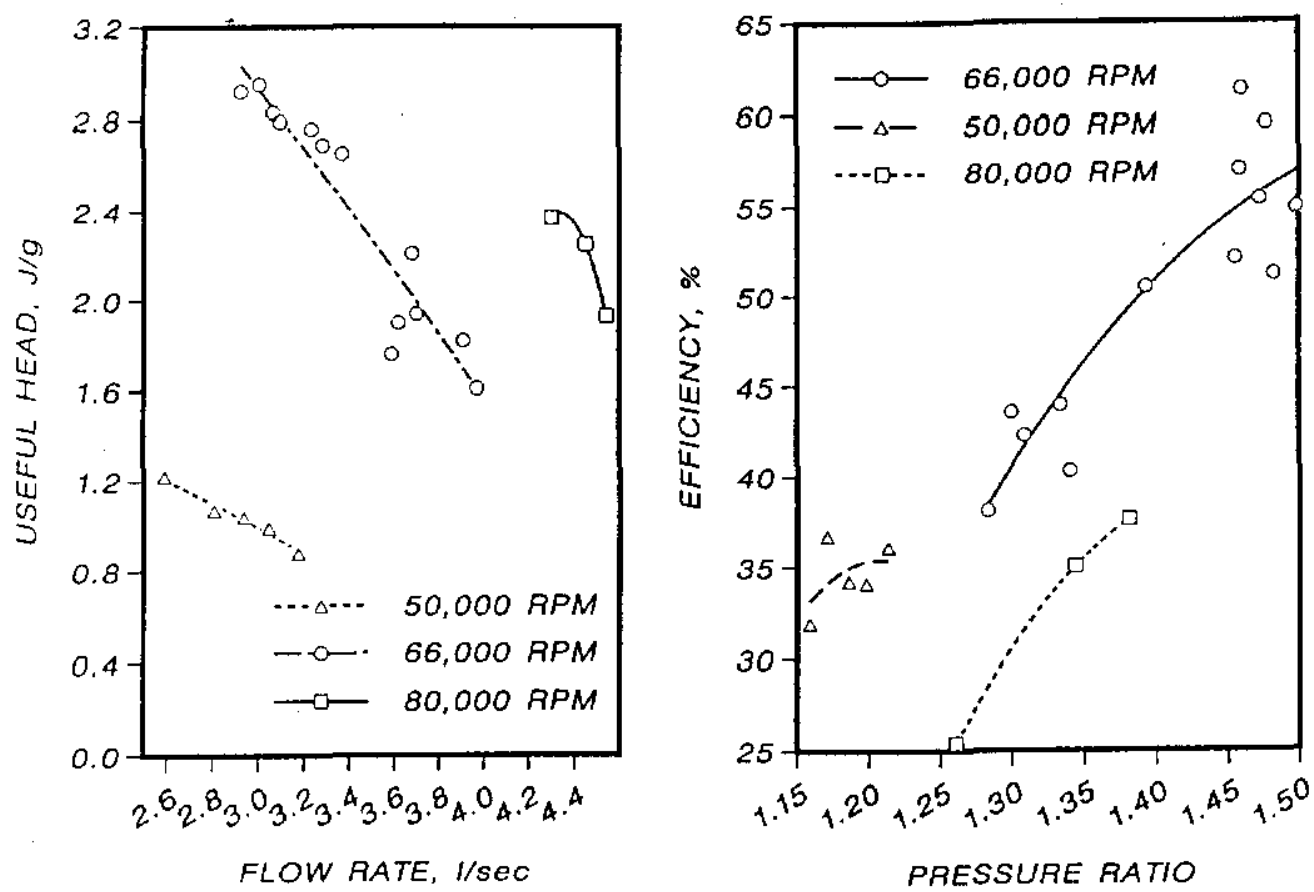

Fig. 3. Creare cold compressor performance.

\section{COLD COMPRESSOR}

\section{Description}

Cryogenic Consultants, Inc. has developed a reciprocating pump/compressor which was delivered to Fermilab in September 1986 for tests. This cold compressor has not been described previously in the literature; therefore a brief description follows. The machine consists of a single stainless steel piston/cylinder arrangement with all-metal spring loaded poppet-style inlet and exhaust valves. The piston shaft extends through a shaft seal in the cover plate to a connecting rod, flywheel, and electric variable speed drive. There are no cams or pull- or push-rods; the valves act as check valves. Some dimensions and specifications are given in Table 3 , with Table 4 summarizing the range of test conditions and Table 5 listing some typical data points.

Table 3. CCI Cold Gas Pump, Model No, CCI-CGP406X300

Bore:

Stroke:

Cylinders:

Piston area:

Displacement:

Pump speed:

Motor:

Speed controller:
4.375 in. $(11.113 \mathrm{~cm})$

3.000 in. $(7.62 \mathrm{~cm})$

One (1)

15.0 in. $\left(97 \mathrm{~cm}^{2}\right)$

45.1 in. $^{3}(739 \mathrm{cc})$

$100-470 \mathrm{rpm}$

1-1/2 hp DC TEFC Motor, 180 VDC

Armature, $1750 \mathrm{rpm}$

$240 \mathrm{~V}, 60 \mathrm{~Hz}, 1$ phase (Input) for

$1-1 / 2$ hp DC Motor, with Run, Stop,

Variable Speed, Local Control 
Speed range tested:

Mass flows:

Pressure ratios:

Inlet pressures:

Adiabatic efficiency range:
250 to $450 \mathrm{rpm}$

0 to $65 \mathrm{~g} / \mathrm{s}$

1.15 to 2.16

1.19 to 0.55 ata

40 to $70 \%$

\section{Test Results}

The CCI cold compressor was run almost continuously from October 1 , 1986 until mid-December 1986. Adiabatic efficiencies ranged from $15 \%$ to $30 \%$ although mechanically the valve seals, piston seal and piston shaft seal all performed well. CCI concluded that the valves were undersized and that the low efficiency was caused by extra work done in overcoming valve pressure drops.

The compressor was taken off line, warmed up, and given a new cylinder, piston, and head assembly with larger valves (provided by CCI). The reconditioned unit was tested and exhibited efficiencies between 40 and 70 percent (including a tentative heat leak estimate of $35 \mathrm{~W} \pm 10 \mathrm{~W}$ ). Efficiency at 1 atm inlet, 1.35 atm exhaust with $49 \mathrm{~g} / \mathrm{s}$ of flow (approximately the Creare design point) was $51 \%$, with higher efficiencies occuring at higher pressure ratios (figure 4). With a 0.7 atm inlet pressure and a mass flow of $48 \mathrm{~g} / \mathrm{s}$ (close to our requirement of $0.7 \mathrm{~atm}, 52 \mathrm{~g} / \mathrm{s}$ for the upgrade to $1 \mathrm{TeV}$ ) an efficiency of $59 \%$ was measured. However, at the speed of 450 RPM required to obtain that mass flow and inlet pressure, mechanical reliability will need to be proven.

Two-phase helium at the inlet created no difficulty. In fact, inlet pressures dropped under these conditions although efficiency could not be measured. One notable achievement was the production of a 1.6 psia inlet condition (2.5K in the 400 liter dewar) achieved by valving off the dewar inlet. Although mass flow could not be determined, exaust pressure was 1.2 atm providing a 10:1 pressure ratio.

Intermittently, during February and March 1987 the CCI unit operated at the Magnet Test Facility to serve as a cold compressor for some 3.0 to 3.5K SSC magnet tests. At MTF the unit demonstrated consistent efficiency with high pressure ratios and low mass flow rates $(<10 \mathrm{~g} / \mathrm{s})$.

\section{Table 5. CCI Cold Compressor: Five Representative Test Results}

Inlet press., atm. abs.

Inlet temp., $\mathrm{K}$ (TIIN)

Inlet enthalpy, $\mathrm{J} / \mathrm{g}$

Inlet entropy, $\mathrm{J} / \mathrm{gK}$

Exhaust press., atm. abs.

Exhaust temp. K (TROT)

Ideal exhaust enthalpy, $\mathrm{J} / \mathrm{g}$

Exhaust enthalpy, $\mathrm{J} / \mathrm{g}$

Mass flow, $\mathrm{g} / \mathrm{s}$

Compressor speed, rpm

Pressure ratio

Adiabatic efficiency \%

$\begin{array}{ccccc}0.55 & 0.92 & 1.04 & 1.19 & 0.71 \\ 3.94 & 4.20 & 4.32 & 4.46 & 4.12 \\ 31.85 & 30.72 & 30.63 & 30.45 & 31.89 \\ 9.654 & 8.548 & 8.348 & 8.117 & 9.245 \\ 1.19 & 1.33 & 1.35 & 1.37 & 1.34 \\ 6.00 & 5.10 & 4.99 & 4.86 & 5.86 \\ 38.00 & 33.21 & 32.34 & 31.32 & 36.86 \\ 41.80 & 34.98 & 33.96 & 32.67 & 40.29 \\ 28 & 43 & 49 & 49 & 48 \\ 376 & 300 & 297 & 250 & 450 \\ 2.16 & 1.45 & 1.30 & 1.15 & 1.90 \\ 61.8 & 58.6 & 51.4 & 39.2 & 59.1\end{array}$




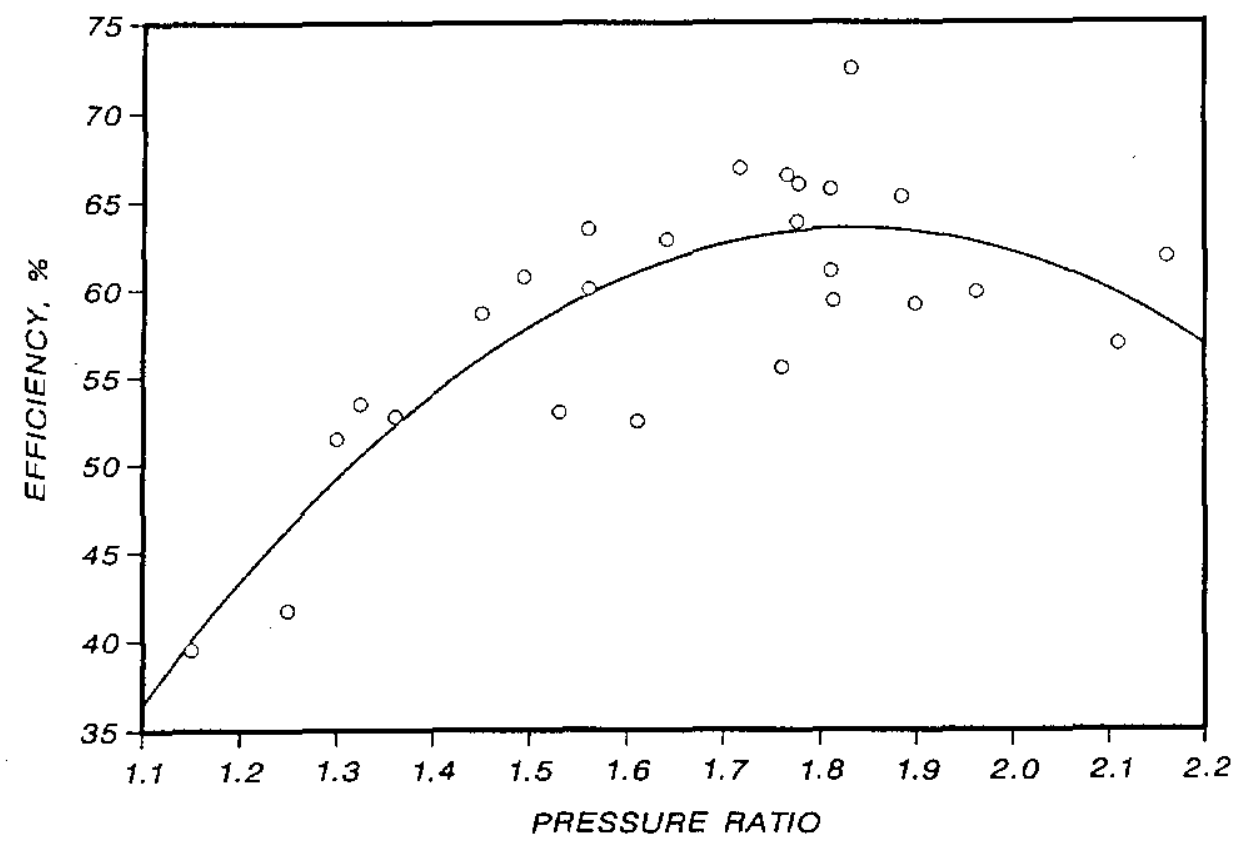

Figure 4. CCI cold compressor performance.

\section{CONCLUSIONS AND PLANS}

Two fundamentally different cold compressors have been tested under similar operating conditions at Fermilab. The CCI reciprocating machine was tested over a larger range of conditions, including subatmospheric inlet pressures.

The Creare unit performed as predicted at its design point $60 \%$ efficiency, $55 \mathrm{~g} / \mathrm{s}$ flow for $1.0 \mathrm{~atm}$ saturated vapor intake and 1.4 atm exhaust). Its gas bearings and lack of valves or mechanical seals give it the advantages of other turbomachinery: potentially long life and low maintenance requirements. Disadvantages include the inability to handle liquid-gas mixtures and a more limited range of flow rates and pressure ratios. Further plans for the Creare unit consist of additional testing with the inclusion of either an integral phase separator/cryostat or a separate phase separator dewar in series upstream of the machine. We are anxious to see how the Creare performs under subatmospheric inlet conditions and higher pressure ratios; these data points are crucial to our evaluation of this unit and should not be difficult to obtain provided there exists adequate phase separation capability.

The CCI cold compressor is still only marginally acceptable from an efficiency standpoint ( $51 \%$ for 1.0 atm intake, 1.35 atm exhaust with $49 \mathrm{~g} / \mathrm{s}$ flow, $59 \%$ with the 0.7 atm intake and $48 \mathrm{~g} / \mathrm{s}$ for our proposed upgrade). However, the machine functions well under two-phase inlet conditions and its versatility permitted operation almost as a cold "vacuum pump" for service at very low mass flows (less than $10 \mathrm{~g} / \mathrm{s}$ ) and high pressure ratios. So far it has demonstrated excellent reliability for its 2500 hours of operation, with valves and seals remaining remarkably leak tight. Future plans call for monitoring mechanical performance over many hours of continuous operation 
at speeds of over 400 RPM. It would also be desirable to better understand the. sources of inefficiency and make further improvements.

Our long-term goal is to install prototype cold compressors of both designs in a few selected satellite refrigerator buildings in order to gain real operating experience with them. Since virtually all operation is remotely overseen from Fermilab's main control room, these machines must prove their controlability and reliability under "instrument only" supervision. Such a scenario will also provide magnet temperature data relevant to a cold compressor's actual ability to increase maximum Tevatron energy.

\section{ACKNOWLEDGEMENTS}

Wayne Stuber, of Air Products, Inc., performed valuable computer simulations while working at Fermilab as a visiting engineer during the summer of 1986. Dodd Stacy and John White of Creare, Inc., provided valuable assistance in testing the Creare cold compressor, and Clyde Harmes of CCI, Inc., has been especially prompt first in delivering the machine to us and then in delivering spare and improved parts.

\section{REFERENCES}

1. J. Theilacker et al, Control of the Tevatron satellite refrigeration system, in: "Advances in Cryogenic Engineering" Vol. 29, Plenum Press, New York (1984), p. 437.

2. M. Kuchnir, Pulsed current resistance thermometry, in: "Advances in Cryogenic Engineering", Vol. 29, Plenum Press, New York (1984), p. 879.

3. T. Jasinski, et al, A generic pump/compressor design for circulation of cryogenic fluids, in: "Advances in Cryogenic Engineering", Vol. 31, Plenum Press, New York (1986), p. 991.

4. W.D. Stacy, Performance test results for a cold helium compressor, in: "AIChE Symposium Series, No. 251", Vol. 82, American Institute of Chemical Engineers, New York (1986), p. 45.

5. W. Swift, H. Sixsmith and A. Schlafke, A small centrifugal pump for circulating cryogenic helium, in: "Advances in Cryogenic Engineering", Vol. 27, Plenum Press, New York (1982), p. 777. 